\title{
ARTICLE OPEN \\ Uncertainty equality with quantum memory and its experimental verification
}

\author{
Hengyan Wang ${ }^{1,2,3,4}$, Zhihao Ma ${ }^{5}$, Shengjun Wu ${ }^{6}$, Wenqiang Zheng ${ }^{7}$, Zhu Cao ${ }^{8}$, Zhihua Chen ${ }^{7}$, Zhaokai $\mathrm{Li}^{1,3,4}$, Shao-Ming Fei ${ }^{9,10}$, \\ Xinhua Peng $\mathbb{D}^{1,3,4}$, Vlatko Vedral ${ }^{11,12}$ and Jiangfeng $\mathrm{Du}^{1,3,4}$
}

As a very fundamental principle in quantum physics, uncertainty principle has been studied intensively via various uncertainty inequalities. A natural and fundamental question is whether an equality exists for the uncertainty principle. Here we derive an entropic uncertainty equality relation for a bipartite system consisting of a quantum system and a coupled quantum memory, based on the information measure introduced by Brukner and Zeilinger (Phys. Rev. Lett. 83:3354, 1999). The equality indicates that the sum of measurement uncertainties over a complete set of mutually unbiased bases on a subsystem is equal to a total, fixed uncertainty determined by the initial bipartite state. For the special case where the system and the memory are the maximally entangled, all of the uncertainties related to each mutually unbiased base measurement are zero, which is substantially different from the uncertainty inequality relation. The results are meaningful for fundamental reasons and give rise to operational applications such as in quantum random number generation and quantum guessing games. Moreover, we experimentally verify the measurement uncertainty relation in the presence of quantum memory on a five-qubit spin system by directly measuring the corresponding quantum mechanical observables, rather than quantum state tomography in all the previous experiments of testing entropic uncertainty relations.

npj Quantum Information (2019)5:39; https://doi.org/10.1038/s41534-019-0153-z

\section{INTRODUCTION}

The uncertainty principle is one of the most important principle in quantum physics. It implies the impossibility of simultaneously determining the definite values of incompatible observables. The more precisely an observable is determined, the less precisely a complementary observable can be known. Based on the distributions of measurement outcomes, the quantum uncertainty relations can be described in various ways; see for instance refs. ${ }^{1-13}$

The uncertainty principle was first formulated via the standard deviation of a pair of complementary observables, known as the Heisenberg's uncertainty principle ${ }^{1} \Delta x \Delta p \geq \hbar / 2$ for the coordinate $x$ and the momentum $p$ in an infinite dimensional Hilbert space. Later the Robertson-Schrödinger uncertainty inequality ${ }^{2,3}$ presented an uncertainty relation for two arbitrary observables in a finite dimensional Hilbert space. Instead of the standard deviation of observables, the uncertainty principle can also be elegantly formulated in terms of entropies related to measurement bases. When a quantum system is projected onto a certain basis $\left\{\left|i_{\theta}\right\rangle \mid i=1\right.$, $2, \cdots, d\}$, where $\theta$ labels the measured observable, the uncertainty of the measurement results has been characterized by the Shannon entropy $H_{\theta}=\sum_{i=1}^{d}-p_{i} \log _{2} p_{i}$, where $p_{i}$ is the probability to obtain the $i$ th basis state $\left|i_{\theta}\right\rangle$. The larger the Shannon entropy $H_{\theta}$ is, the more uncertain the measurement results are. In terms of the
Shannon entropies of the measurement results, the uncertainty principle can be formulated as $H_{\theta}+H_{\tau} \geq \log _{2} \frac{1}{c} \cdot{ }^{4}$ Here $1 / c$ quantifies the degree of complementarity of two observables $\theta$ and $\tau$.

The above uncertainty relations only concern a single quantum system. By taking the entanglement with a memory system into account, ${ }^{14}$ an entropic uncertainty relation in the presence of quantum memory has been investigated in ref. ${ }^{15}$. It has been shown that for a bipartite state $\rho_{A B}$, performing measurements on one of the subsystems $A$ gives rise to the following relation

$S(\theta \mid B)+S(\tau \mid B) \geq \log _{2} \frac{1}{c}+S(A \mid B)$,

where $S(A \mid B)$ and $S(\theta \mid B)(S(\tau \mid B))$ denote, respectively, the conditional von Neumann entropies of the initial bipartite state $\rho_{A B}$ and the final bipartite state $\rho_{\theta B}\left(\rho_{T B}\right)$ after the measurement in the basis $\left\{\left|i_{\theta}\right\rangle\right\}\left(\left\{\left|i_{\tau}\right\rangle\right\}\right)$. This uncertainty relation was further extended to the smooth entropy case. ${ }^{16}$ With considering the entangled quantum memory, these uncertainty relations have potential applications in entanglement witnessing and quantum key distributions (QKD). ${ }^{15,17,18}$ However, the above results all concern measurements only on two observables and are given in inequality forms.

In this work, we consider projective measurements based on mutually unbiased bases (MUBs). ${ }^{19-24}$ The MUB measurements are complementary to each other in the sense that any pair of bases

\footnotetext{
${ }^{1}$ Hefei National Laboratory for Physical Sciences at the Microscale and Department of Modern Physics, University of Science and Technology of China, 230026 Hefei, China; ${ }^{2}$ Department of Physics, Zhejiang University of Science and Technology, 310023 Hangzhou, China; ${ }^{3}$ CAS Key Laboratory of Microscale Magnetic Resonance, University of Science and Technology of China, $230026 \mathrm{Hefei}$, China; ${ }^{4}$ Synergetic Innovation Center of Quantum Information and Quantum Physics, University of Science and Technology of China, 230026 Hefei, China; ${ }^{5}$ School of Mathematical Sciences, Shanghai Jiaotong University, 200240 Shanghai, China; ${ }^{6}$ Kuang Yaming Honors School, Nanjing University, 210023 Nanjing, Jiangsu, China; ${ }^{7}$ College of Science, Zhejiang University of Technology, 310023 Hangzhou, Zhejiang, China; ${ }^{8}$ Center for Quantum Information, Institute for Interdisciplinary Information Sciences, Tsinghua University, 100084 Beijing, China; ${ }^{9}$ School of Mathematical Sciences, Capital Normal University, 100048 Beijing, China; ${ }^{10}$ MaxPlanck-Institute for Mathematics in the Sciences, 04103 Leipzig, Germany; ${ }^{11}$ Atomic and Laser Physics, University of Oxford, Clarendon Laboratory, Parks Road, OX1 3PU Oxford, United Kingdom and ${ }^{12}$ Centre for Quantum Technologies, National University of Singapore, 3 Science Drive 2, 117543 Singapore, Singapore

Correspondence: Xinhua Peng (xhpeng@ustc.edu.cn) or Jiangfeng Du (djf@ustc.edu.cn)

These authors contributed equally: Hengyan Wang, Zhihao Ma, Shengjun Wu
}

Received: 19 November 2018 Accepted: 11 April 2019

Published online: 17 May 2019 
are maximally unbiased. They are deeply connected to the Born's principle of complementarity ${ }^{21}$ and closely related to the waveparticle duality. ${ }^{25,26} \mathrm{~A}$ complete set of MUBs consists of at most $d$ +1 observables, where $d$ is the dimension of the state space. Comparing with the incomplete case, the advantage of a complete set of MUB measurements is informatively complete com $^{23,24}$ and meaningful in quantum information progressing. ${ }^{21}$ It is therefore not surprising that a complete set of MUB measurements is crucial in entanglement detection. ${ }^{27,28}$ It was also proved that using a complete set of MUBs is much better than using two observables in QKD. ${ }^{19}$

In ref. ${ }^{6}$ an entropic uncertainty relation involving $d+1$ MUB measurements has been obtained in terms of von Neumann entropy. However, it only dealt with a single system (in this case the von Neumann entropy is just the Shannon entropy of the measurement probability distributions), and the uncertainty relation is given by an inequality. In fact, the Shannon entropy is a natural measure of our ignorance regarding the properties of a classical system, because in classical measurements the observation removes our ignorance about the state by revealing the properties of the system which are considered to be pre-existing and independent of the observation. In contrast to classical measurements, one cannot say that quantum measurements reveal a pre-existing property of a quantum system. Therefore, the Shannon entropy could be thought of as "conceptually" inadequate in quantum physics. ${ }^{29}$ In ref. ${ }^{26}$ the authors proposed a new measure of quantum information, which takes into account that the only features of quantum systems known before a measurement are the probabilities for various events to occur. It has significant physical meaning and various applications in quantum information processing such as quantum randomness, quantum state estimation, quantum teleportation and quantum metrology. ${ }^{30-36}$ Moreover, a series of works have been shown, together with many applications, that in single quantum system, the sum of the individual measures of information for MUBs is invariant under the choice of the particular set of complementary observations and conserved if there is no information exchange with environments. ${ }^{26,29-34,37-42}$

In this article, we adopt the information measure proposed in ref. ${ }^{26}$ and consider the uncertainty relation in the presence of quantum memory. Interestingly, we find that if we take a complete set of MUB measurements into account, we can obtain an uncertainty equality that the sum of measurement uncertainties over all MUBs on a subsystem in the presence of quantum memory is equal to a fixed quantity determined by the initial state. It gives rise to a kind of conservation relation of the uncertainties related to these MUB measurements. We further show the elegant applications of our result in quantum guessing game and quantum random number generation. We also experimentally verify this uncertainty equality by directly measuring the uncertainties on a nuclear spin system. Our method avoids the tomography process and allows one to perform verification experiments in large quantum systems.

\section{RESULTS}

Measurement of uncertainty relations

Let $\left(p_{1}, p_{2}, \ldots, p_{d}\right)$ be the probabilities for the $d$ measurement outcomes. The lack of information about the $j$ th outcome with respect to a single experimental trial is given by $p_{j}\left(1-p_{j}\right)$. The total lack of information regarding all $d$ possible experimental outcomes is then given by $\sum_{j=1}^{d} p_{j}\left(1-p_{j}\right)=1-\sum_{j=1}^{d} p_{j}^{2}$, which is minimal if one probability is equal to a unity and maximal if all the probabilities are equal. In fact, $1-\sum_{j=1}^{d} p_{j}^{2}$ is nothing but $1-\mathrm{Tr}$ $\left(\rho^{2}\right)$, where $\rho$ is the state after a quantum (projective) measurement, the linear entropy of the measured state. Therefore, the lack of information regarding all $d$ possible experimental outcomes can be described by the linear entropy of a $d$-level quantum state $\rho, S_{L}(\rho)=1-\operatorname{Tr}\left(\rho^{2}\right) . S_{L}(\rho)$ ranges from 0 (when $\rho$ is a pure state) to $(d-1) / d$ (when $\rho$ is maximally mixed). Unlike that in ref. ${ }^{26}$, here we do not introduce a normalization factor to have a range between 0 and $\log _{2} d$, so the measure of uncertainty in terms of linear entropy does not have the unit of a "bit". However, it quantifies uncertainty in a natural way: an uncertainty of 0 means the outcome is $100 \%$ certain while an uncertainty approaching 1 means the outcome is almost random.

For a bipartite state $\rho_{A B}$ in a $d \times D(D \geq d)$ dimensional composite Hilbert space, if system $A$ is projected on to the basis $\left\{\left|i_{\theta}\right\rangle \mid i=1,2\right.$, $\cdots, d\}$, the overall state of the composite system after the nonselective measurement ${ }^{43,44}$ on $A$ is given as

$\rho_{\theta B}=\sum_{i=1}^{d}\left|i_{\theta}\right\rangle_{A}\left\langle i_{\theta}\right| \otimes_{A}\left\langle i_{\theta}\left|\rho_{A B}\right| i_{\theta}\right\rangle_{A}$.

We can introduce the conditional linear entropy

$S_{L}(\theta \mid B):=S_{L}\left(\rho_{\theta B}\right)-S_{L}\left(\rho_{B}\right)=\operatorname{Tr}\left(\rho_{B}^{2}\right)-\operatorname{Tr}\left(\rho_{\theta B}^{2}\right)$

as a measure of the uncertainty about Alice's measurement result given Bob's state, where the reduced state $\rho_{B}=\operatorname{Tr}_{A}\left(\rho_{\theta B}\right)=\operatorname{Tr}_{A}\left(\rho_{A B}\right)$ is independent of the measurement basis. It is straightforward to show that the conditional linear entropy $S_{L}(\theta \mid B)$ is always nonnegative. As an example, suppose $\rho_{A B}$ is a maximally entangled pure state. Alice can perform a measurement on her system in any basis. The possible resulting states of Bob's system are orthogonal to each other, and each possible resulting state is in one-to-one correspondence to Alice's resulting state. Therefore, given Bob's state, Alice's measurement result can be determined with certainty. In this case $S_{L}(\theta \mid B)$ vanishes. If $\rho_{A B}=\sum_{i=1}^{d} \sqrt{\lambda_{i}}|i i\rangle$ is a partially entangled state written in its Schmidt bases, after Alice measures her system in the Schmidt basis, Bob's possible resulting states are orthogonal to each other and the Alice's measurement result is completely determined without uncertainty when Bob's state is given. This is also confirmed by the vanishing conditional entropy as $S_{L}\left(\rho_{\theta B}\right)=S_{L}\left(\rho_{B}\right)=\sum_{i} \lambda_{i}^{2}$. However, if Alice performs a measurement on a basis that is not the Schmidt basis, the possible resulting states of Bob's system are not orthogonal and cannot be distinguished with certainty, and thus uncertainty of Alice's measurement result exists even when Bob's state is known. This fact is again confirmed by the observation that the conditional linear entropy is strictly greater than zero in this case. The conditional linear entropy is thus a good measure of the uncertainty about Alice's measurement result given Bob's state. It depends on the basis in which the measurement is performed in general. When Alice tries to find a basis to perform the measurement on her system so that Bob will know her result with minimum uncertainty, then using another MUB to perform the measurement will result in Bob having a large uncertainty about Alice's result. However, the whole uncertainty running over all possible MUB measurements is fixed. This uncertainty relation is formulated in the following theorem (the proof involves subtle mathematical techniques, see Method $A$ ).

Theorem For any density matrix $\rho_{A B}$ on a composite Hilbert space $H_{A} \otimes H_{B}$ of dimension $d \times D$, we have the following uncertainty equality

$\sum_{\theta=1}^{d+1} S_{L}(\theta \mid B)=d\left(\operatorname{Tr}\left(\rho_{B}^{2}\right)-\frac{1}{d} \operatorname{Tr}\left(\rho_{A B}^{2}\right)\right)$

when a complete set of $d+1$ MUBs exists for the $d$-dimensional Hilbert space $H_{A}$.

The theorem shows that the total uncertainty related to the measurements over all $d+1$ MUBs of a subsystem is exactly given by a fixed quantity, $d \operatorname{Tr}\left(\rho_{B}^{2}\right)-\operatorname{Tr}\left(\rho_{A B}^{2}\right)$, which is determined only by the initial bipartite state. This quantity is always nonnegative and can be viewed as the total measurement uncertainty of a 
subsystem, given the state of the other subsystem. Different from the uncertainty inequality (1) based on the von Neumann entropy, here we obtain the equality (4). Note that this equality (4) is also completely different from the one given in ref. ${ }^{45}$ which is based on only ONE positive operator-valued measure consisting of uniformly all the measurement operators of $d+1$ MUBs (see Remark in Method A). In general, when there are only M MUBs available or when we are only interested in certain M MUBs, we always have the following uncertainty inequality,

$$
\sum_{\theta=1}^{M} S_{L}(\theta \mid B) \geq(M-1)\left(\operatorname{Tr}\left(\rho_{B}^{2}\right)-\frac{1}{d} \operatorname{Tr}\left(\rho_{A B}^{2}\right)\right) .
$$

With each additional MUB, the lower bound of total uncertainty is increased by a fixed amount $\operatorname{Tr}\left(\rho_{B}^{2}\right)-\frac{1}{d} \operatorname{Tr}\left(\rho_{A B}^{2}\right)$.

To illustrate the implications of the theorem, let us consider that Alice and Bob are both users of quantum technology. In order to make a hard decision on whether she should accept Bob's invitation to see a film, Alice asks Bob to send her a qubit $A$. Alice can measure the qubit with the three (Pauli) observables $\sigma_{x}, \sigma_{y}$ and $\sigma_{z}$ at her choice. After the measurement, Alice announces her choice of the observable, and Bob is supposed to guess the Alice's measurement results. Alice would accept (deny) Bob's request if his guess is correct (wrong). Bob tries to gain Alice's acceptance by entangling the qubit $A$ with his local qubit $B$ in the preparation stage. From the theorem, we know that the sum of uncertainties (of Bob's guess at Alice's measurement results given the state of $B$ ) in three different cases is equal to the quantity $Q=2 \operatorname{Tr}\left(\rho_{B}^{2}\right)-$ $\operatorname{Tr}\left(\rho_{A B}^{2}\right)$ that is completely determined by the initial state $\rho_{A B}$. Bob can minimize the quantity $Q$ by preparing an EPR state, thus win Alice's acceptance with certainty, a result that cannot be obtained from an uncertainty inequality like the one based on Shannon entropy (see Fig. 1).

On the practical side, the theorem also provides possible applications in quantum random number generation, especially semi-self-testing quantum random number generators (QRNGs) which are more robust to device imperfections. In a typical setup of a semi-self-testing QRNG, ${ }^{46-48}$ Alice and Bob share a quantum state $\rho_{A B}$, e.g., an EPR pair. If both parties are trusted, the measurement outcome of one party will be random to the other

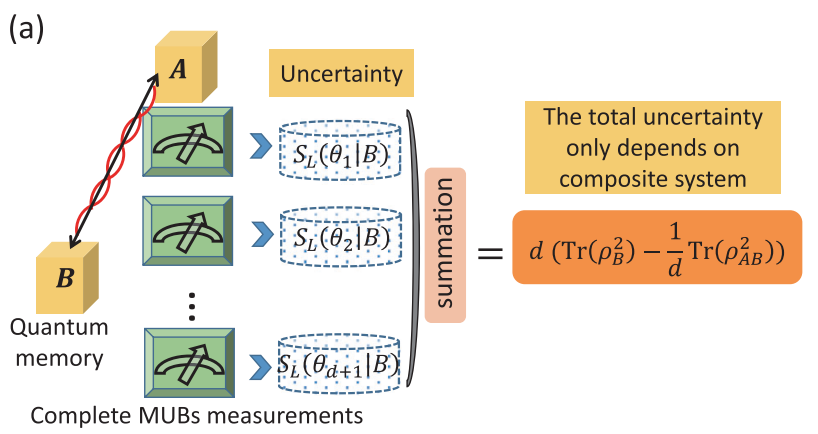

(b)

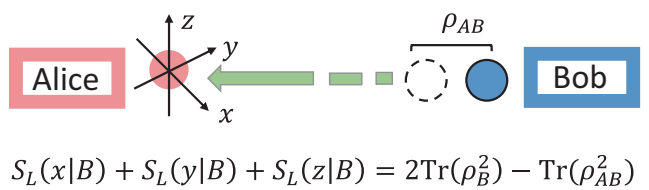

Fig. 1 a Sketch of the proposal. $\mathbf{b}$ Illustration of implications of the theorem. Alice chooses to measure one of the three Pauli matrices $\sigma_{x}, \sigma_{y}$ and $\sigma_{z}$ on qubit $A$, and then informs Bob her choice and requests him to guess her measurement outcome. In order to guess Alice's measurement outcome with less uncertainty, Bob can entangle qubit $A$ with a local qubit $B$ before sending qubit $A$ to Alice, so that to minimize the uncertainty party when Alice measures in the computational basis and Bob measures in the diagonal basis. However, if one of the parties is corrupted, e.g., due to device imperfections, this scheme is broken. To show this, consider that one party switches to the same basis as the other party. A common solution is that each party randomly uses multiple basis, such as $\sigma_{x}, \sigma_{y}$ or $\sigma_{z}$ basis. ${ }^{46-48}$ Now, we consider the following semi-self-testing scenario. Alice first chooses a reference frame, and randomly performs measurements in one of the MUB basis. The reference frame is assumed to be reliably chosen, but Alice does not have a free will to randomly choose her measurement basis, i.e., the basis choice may be manipulated by an adversary who wishes to corrupt Alice's randomness, such as Bob. Hence, the entropy of Alice's random outcomes with respect to Bob is the smallest entropy $S_{L}(\theta \mid B)$ among all measurement choices. To maximize this quantity, the theorem shows that $S_{L}(\theta \mid B)$ should be equal for all $\theta$ s. Thus, the maximum entropy of a semi-self-testing QRNG is $\left[d \operatorname{Tr}\left(\rho_{B}^{2}\right)-\operatorname{Tr}\left(\rho_{A B}^{2}\right)\right] /(d+1)$. This limit on semi-self-testing QRNGs also cannot be obtained from an uncertainty inequality. Finally, note that entanglement-based QRNGs considered here have a higher randomness generation rate compared to prepare-andmeasure QRNGs, ${ }^{49}$ and cannot be analyzed by using the tools developed by Brukner and Zeilinger. ${ }^{26}$

\section{Experimental verification}

To experimentally investigate the uncertainty conservation, a twoqubit system $\rho_{A B}$, chosen as the test system, is prepared in the following states:

$\rho_{A B}(a, x)=x\left|\psi_{a}\right\rangle\left\langle\psi_{a}\right|+\frac{1-x}{4} l_{4}$,

where $\left|\psi_{a}\right\rangle=\cos (\alpha / 2)|01\rangle-\sin (\alpha / 2)|10\rangle$. These states are mixed states composed of one pure state with weight $x$ and the maximal mixed state with weight $(1-x) / 4$. The parameters $a$ characterizes the entanglement of the pure part and $x$ characterizes the purity of the state. When $a=\pi / 2$ and $x=1$, the bipartite state is one of the Bell states. The other three Bell states can be obtained by local unitary operations while the linear entropy remains invariant under such transformations.

The key part of the experiments is to measure the system's (conditional) linear entropy. Similar to the measurement of von Neumann entropy in previous experiments, ${ }^{50,51}$ linear entropy can be indirectly measured by full quantum state tomography. ${ }^{52}$ However, this is inefficient for large-size quantum systems. Since the linear entropy is directly related to the purity $\operatorname{Tr}\left(\rho^{2}\right)$ that can be directly obtained by $\operatorname{Tr}\left(\rho^{2}\right)=\operatorname{Tr}\left(V_{2} \rho \otimes \rho\right)^{53}$ with a copy of $\rho$, this allows us to employ an operational and direct way to experimentally verify the uncertainty conservation relation. Here the operator $V_{2}$ is the SWAP operation, i.e., $V_{2}\left|\psi_{1} \psi_{2}\right\rangle=\left|\psi_{2} \psi_{1}\right\rangle$, that exchanges the states of two subsystems. By using one ancillary probe qubit to perform the interferometric measurement, we can directly obtain all the required information of the purities from the probe qubit, as shown in Fig. 2 a.

Physical system. To verify the equation in the experiments, we used the sample named 1-bromo-2,4,5-trifluorobenzene as a fivequbit NMR quantum system which consists of two ${ }^{1} \mathrm{H}$ spins and three ${ }^{19} \mathrm{~F}$ spins, dissolved in the liquid-crystal $\mathrm{N}$-(4-methoxybenzylidene)-4-butylaniline (MBBA). Spins $\mathrm{H}_{3}$ and $\mathrm{H}_{4}$ are labeled as the bipartite system $\rho_{A B}$, spins $F_{1}$ and $F_{2}$ as the copy system $\rho_{A^{\prime} B^{\prime}}$, and spin $F_{5}$ as the probe qubit $\rho_{\text {probe. }}$ The effective Hamiltonian of the five-qubit system in double rotating frame is

$H_{\mathrm{NMR}}=\sum_{i=1}^{5} \pi \nu_{i} \sigma_{z}^{i}+\sum_{1 \leq j<k \leq 5} \frac{\pi}{2}\left(J_{j k}+2 D_{j k}\right) \sigma_{z}^{j} \sigma_{z}^{k}$,

where $\sigma_{z}$ is the Pauli operator, $v_{i}$ is the chemical shift of spin- $i$ and $J_{j k}+2 D_{j k}$ is the effective coupling constant of spin- $j$ and spin- $k$. 
(a)

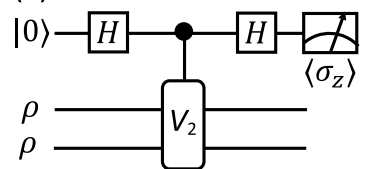

(b)

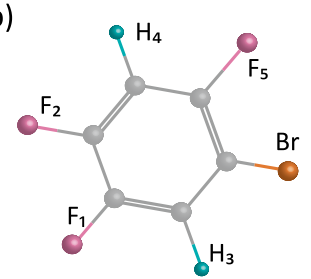

(c)

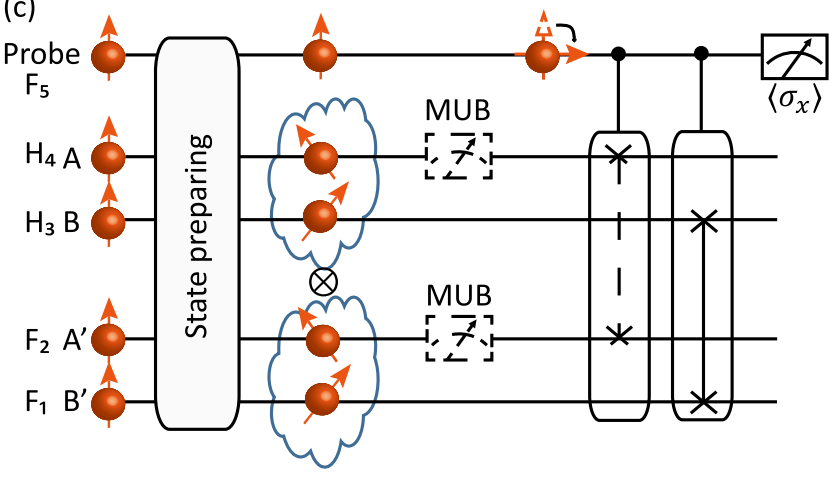

Fig. 2 a Quantum circuit of directly measuring the purity. b Molecular structure for NMR quantum register. c Experimental schematic for verifying the measurement uncertainty relation. MUB measurements are performed on subsystem $A$ to get $\rho_{\theta B}$, and meanwhile the same process is applied on the mirror subsystem $A^{\prime}$. Then controlled-SWAP gates are performed for the measurements of the linear entropies $S_{L}(\theta \mid B)$ in LHS of Eq. (4). The purity information on the original state $\rho_{A B}$, i.e., the RHS of Eq. (4), are obtained without MUB measurement in the dashed lines. The purities of the bipartite system $A B: \operatorname{Tr}\left(\rho_{A B}^{2}\right)$ and $\operatorname{Tr}\left(\rho_{\theta B}^{2}\right)$, are obtained by two controlled-SWAP gates $C_{\text {swap }}$ applying on subsystems $A A^{\prime}$ and $B B^{\prime}$ while only one $C_{\text {swap }}$ is operated on subsystem $B B^{\prime}$ (denoted by the solid line) for the purity of $\operatorname{subsystem} \operatorname{Tr}\left(\rho_{B}^{2}\right)$ and $\operatorname{Tr}\left(\rho_{B \mid \theta}^{2}\right)$, where $\rho_{B \mid \theta}=\operatorname{Tr}_{A}\left(\rho_{\theta B}\right)$

The molecular structure is shown in Fig. $2 \mathrm{~b}$, and the relevant parameters are shown in Method B.

Experimental procedure. Figure $2 \mathrm{c}$ shows the experimental schematic for verifying the measurement uncertainty relations. It can be divided into four parts.

1. Preparing initial state. From thermal equilibrium state, we first initialized the system to a labeled pseudo-pure state (LPPS) $\quad \rho_{\text {LPPS }}=\frac{1}{32} l_{32}+\varepsilon \sigma_{z}^{\text {probe }} \otimes|0000\rangle_{A B A / B \prime}\langle 0000|$ with selective-transition method, ${ }^{54}$ where $\varepsilon \approx 10^{-5}$ is the polarization and $I_{32}$ is the 32 -dimension identity matrix. There is no dynamical and measurement effect on the part of identity density matrix; thus in the following, we conventionally denote the state with the deviation density matrix, ${ }^{55}$ ignoring the identity matrix. Then the product state $\rho_{A B}(a, x)$ $\otimes \rho_{A^{\prime} B^{\prime}}(a, x)$ was prepared from $|0000\rangle_{A B A^{\prime} B^{\prime}}\langle 0000|$, where $\rho_{A^{\prime}}$ $B^{\prime}$ is the copy of $\rho_{A B}$. We vary the weight $x$ by rotation with different angles and a following nonunitary gradient pulse. The details of initialization process are shown in Method C.

2. Performing MUB measurements. The complete MUB measurements were implemented on subsystem $A$. For a twodimensional system, the simplest case of MUBs are

$$
\begin{aligned}
& M_{0}=\{|0\rangle,|1\rangle\}, M_{1}=\left\{\frac{|0\rangle+|1\rangle}{\sqrt{2}}, \frac{|0\rangle-|1\rangle}{\sqrt{2}}\right\}, \\
& M_{2}=\left\{\frac{|0\rangle+i|1\rangle}{\sqrt{2}}, \frac{|0\rangle-i|1\rangle}{\sqrt{2}}\right\} .
\end{aligned}
$$

They are just the eigenvectors of Pauli operators $\sigma_{x}, \sigma_{y}$ and $\sigma_{z}$. In NMR, such MUB projective measurements can be emulated using pulsed magnetic field gradients. ${ }^{56}$ Without interfering the unselected systems ( $B$ and its copy $B^{\prime}$ ), we realized the MUB measurements on subsystem $A$ and its copy $A^{\prime}$ by the gradient echo technology, ${ }^{57}$ i.e., by selective $\pi$ pulses to the other spins, this dephasing operation (i.e., projective measurement of $\sigma_{z}$ ) can be selectively performed on some specific spins. It is in principle necessary to refocus all the evolutions under the internal Hamiltonian during the gradient echo. However, this sequence can be simplified when we only care about the purity of the crashed state. For example, the projective measurement of $M_{0}$ on spin $\mathrm{F}_{2} \quad(A)$ and $\mathrm{H}_{4} \quad\left(A^{\prime}\right)$ are accomplished by $P_{z}^{\mathrm{F}_{2}, \mathrm{H}_{4}}=G_{z}-[\pi]_{X}^{\mathrm{F}_{1}, \mathrm{H}_{3}, \mathrm{~F}_{5}}-G_{z}-[\pi]_{-x}^{\mathrm{F}_{1}, \mathrm{H}_{3}, \mathrm{~F}_{5}}$, where the evolutions under internal effective coupling Hamiltonian related to three qubits $\mathrm{F}_{1}, \mathrm{H}_{3}$ and $\mathrm{F}_{5}$ are reserved during the pulse sequence. However, by some calculations, these undesired evolutions will lead to an error less than $2[1-\cos (2 \Delta \theta)] \approx$ 0.065 on the purity measurements of the subsystem $A$ or $A^{\prime}$, mainly determined by the different evolutions on qubits $F_{1}$, $\mathrm{H}_{3}$ due to the different effective coupling constants $J_{\mathrm{F}_{1}, \mathrm{~F}_{5}}+$ $2 D_{\mathrm{F}_{1}, \mathrm{~F}_{5}}$ and $J_{\mathrm{H}_{3}, \mathrm{~F}_{5}}+2 D_{\mathrm{H}_{3}, \mathrm{~F}_{5}}$. Here $\Delta \theta=\pi\left[\left(J_{\mathrm{F}_{1}, \mathrm{~F}_{5}}+2 D_{\mathrm{F}_{1}, \mathrm{~F}_{5}}\right)-\right.$ $\left.\left(J_{H_{3}, F_{5}}+2 D_{H_{3}, F_{5}}\right)\right] t_{G_{z}} / 2$ with the duration $t_{G_{z}}$ of pulsed magnetic field gradient $G_{z}$. To perform projective measurements of $M_{1}$ and $M_{2}$ on specific spins, we first selectively rotate the spins with $[\pi / 2]_{-y}$ or $[\pi / 2]_{x}$ rotations, then performs the projective measurement of $M_{0}$, e.g., $P_{x}^{\mathrm{F}_{2}, \mathrm{H}_{4}}=$ $[\pi / 2]_{-y}^{\mathrm{F}_{2}, \mathrm{H}_{4}}-P_{z}^{\mathrm{F}_{2}, \mathrm{H}_{4}}$ and $P_{y}^{\mathrm{F}_{2}, \mathrm{H}_{4}}=[\pi / 2]_{x}^{\mathrm{F}_{2}, \mathrm{H}_{4}}-P_{z}^{\mathrm{F}_{2}, \mathrm{H}_{4}} .{ }^{56}$

3. Measuring the purities. After the MUBs on the subsystem $A$ and $A^{\prime}$, performing the quantum circuit in Fig. 2a will give the purity information on the resulting state $\rho_{\theta B}$ after MUBs. For example, when two controlled-SWAP gates $\left(C_{\text {swap }}\right)$ are applied to both subsystems $A A^{\prime}$ and $B B^{\prime}$, one gets the purity of the bipartite system $A B: \operatorname{Tr}\left(\rho_{\theta B}^{2}\right)$; when only one $C_{\text {swap }}$ is applied to the subsystem $B B^{\prime}$, one gets the purity of subsystem $B: \operatorname{Tr}\left(\rho_{B \mid \theta}^{2}\right)$ with $\rho_{B \mid \theta}=\operatorname{Tr}_{A}\left(\rho_{\theta B}\right)$, as shown in Fig. 2c. Likely, the related purities of the original state $\rho_{A B}$ : $\operatorname{Tr}\left(\rho_{A B}\right)$ and $\operatorname{Tr}\left(\rho_{B}^{2}\right)$, are obtained by the similar procedure without the MUBs. It can be noted the initial state of the probe qubit is different from the original method in Fig. 2a. We initialize the probe qubit as $\sigma_{z}^{\text {probe }}$ in our experiment. However, this will not affect the measure of the purity by the quantum circuit $U_{\mathrm{qc}}$ in Fig. 2a, i.e.,

$\operatorname{Tr}\left[\sigma_{z}^{\text {probe }} U_{\mathrm{qc}}\left(\sigma_{z}^{\text {probe }} \otimes \rho \otimes \rho\right) U_{\mathrm{qc}}^{\dagger}\right]=2 \operatorname{Tr}\left[V_{2}(\rho \otimes \rho)\right]=2 \operatorname{Tr}\left(\rho^{2}\right)$.

Moreover, since the direct observable in NMR is $\sigma_{x}$, the final Hadamard gate can be canceled out by the readout operation. Therefore, through integrating the NMR spectra of the probe spin $\mathrm{F}_{5}$, we directly measure the purities on the related states. By calculating the linear entropy, both sides of Eq. (4) are obtained without quantum state tomography.

Experimental results. The experimental results are shown in Fig. 3. As expected, the sum of uncertainties decreases to zero when the bipartite system is in maximally entangled state. With certain $a$, lower purity corresponds to higher uncertainty. From Fig. 3a, b, we can see that the experimental results are in accord with theoretical expectations and the uncertainty conversation relation holds with high precision. Figure $3 c$ shows the final NMR spectra of the probe 
(a)

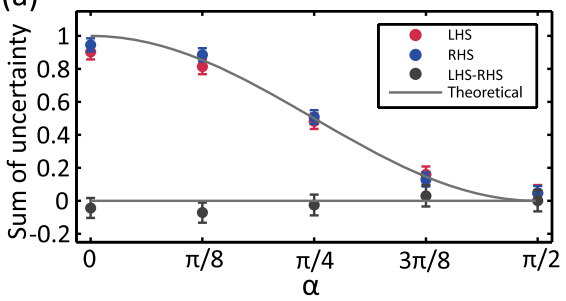

(b)

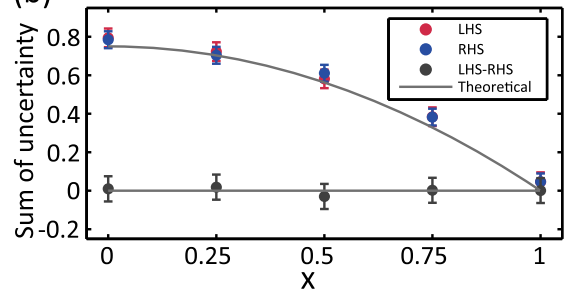

(c)

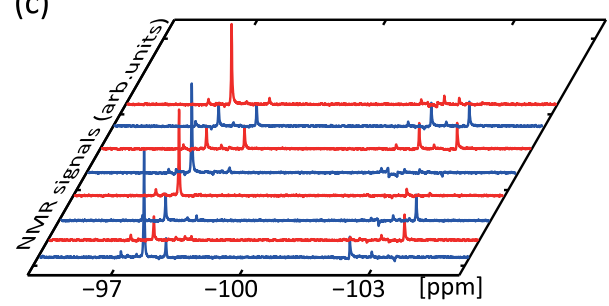

(d)

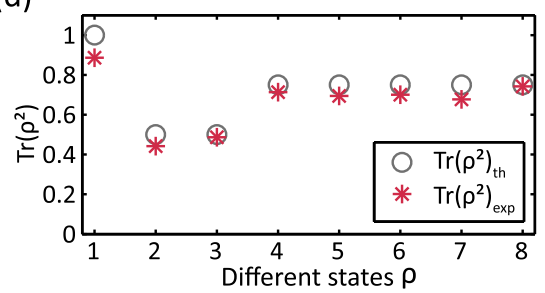

Fig. 3 Experimental results of verifying the measurement uncertainty with the input states $\rho_{A B}(a, 1)(\mathbf{a})$ and $\rho_{A B}(\pi / 2, x)(\mathbf{b})$. Red and blue dots represent the measured values of LHS and RHS of Eq. (4), respectively. The experimental data are rescaled by extracting the decoherence effect, shown by filled squares. The original data are shown in Method E. The dark gray curves are theoretical expectations. The bars are plotted from the infidelity of readout process. c Experimental NMR spectra of probe spin $\left(\mathrm{F}_{5}\right)$ for $\rho_{A B}(\pi / 4,1)$. From bottom to up, the related resulting states after measurement are, respectively, $\rho_{A B}, \rho_{x B}, \rho_{y B}, \rho_{z B}, \rho_{B}, \rho_{B \mid x}, \rho_{B \mid y}, \rho_{B \mid z}$. d Measured purities of the resulting states shown in (c). Each purity is obtained from the sum of the integral values of the corresponding peak in (c)

qubit for one certain initial state $\rho_{A B}(\pi / 4,1)$. The sum of the integral values of all the peaks is read as the purity of the related state in our experiment, as shown in Fig. 3d.

In our experiments, to avoid the error accumulation and alleviate the influence of the decoherence, we used high-fidelity engineered quantum control pulses, which exploit the gradient ascent pulse engineering (GRAPE) algorithm, ${ }^{58}$ to implement the quantum circuit in the experiments. The experiments for pure states contain eight GRAPE pulses with the total duration of about $74 \mathrm{~ms}$, while for mixed states, we used 9 11 GRAPE pulses with total durations of $67-85 \mathrm{~ms}$. We numerically optimized all GRAPE pulses with considering $5 \%$ ratio frequency ( $\mathrm{rf}$ ) field inhomogeneity, so that they are more robust in experiments. All GRAPE pulses used in the experiments have theoretical fidelities above $99.3 \%$. Numerical simulations show that the imperfection of GRAPE pulses causes infidelity of $2-4 \%$ in the final states. Due to the short relaxation times of the liquid-crystal sample, the experiments suffer severe decoherence effect. We numerically simulated the dynamical process and estimated the attenuation factors caused by decoherence effect in the experiments. ${ }^{59,60}$ Then we rescaled the experimental results. The details can be also found in Method E. In the numerical simulations, we found that transverse relaxation time $T_{2}$ plays a leading role in the decoherence process, while the longitude relaxation time $T_{1}$ has little influence. The imperfection in preparing the labeled PPS also causes some errors. The highest unexpected peak in the labeled PPS NMR spectrum of spin $F_{5}$ is about $3 \%$ intensity of the only expected peak.

\section{DISCUSSION}

In conclusion, we have derived a novel entropic measurement uncertainty relation in bipartite systems with a quantum memory. It has been shown that after a complete set of MUB measurements on one partite, the total uncertainty on the other partite is exactly given by the purities of the initial system and the memory. Substantially different from the previous uncertainty relations with inequalities, we presented an equality of uncertainty relation for the case with a quantum memory, which implies direct applications to quantum random number generation and quantum guessing games. Moreover, the relation (4) is independent of the choices of the MUBs. Therefore, the relation (4) gives rise to a kind of conservation of measurement uncertainties, in the sense that (4) is invariant under the transformation of MUBs.

Our theorem gives an uncertainty equality relation for arbitrary dimensional bipartite systems consisting of a quantum system and a coupled quantum memory. It should be emphasized that, even for single-partite systems, it is already quite difficult to obtain an uncertainty equality relation for high-dimensional case. The high-dimensional bipartite case is much more complex than the case of single-partite one. ${ }^{26,29-34,37-42}$ Therefore, as one sees in Method $A$, it is not surprising that the derivation of our uncertainty relations needs subtle mathematical techniques.

With the help of one mirror system of the measured system and one additional probe qubit, we have provided the first experimental verification of this measurement uncertainty relation in an NMR quantum processor, where the experimental data of uncertainty quantities have been directly obtained by measuring the involved entropies without quantum state tomography. This method allows one to perform verification experiments in large quantum systems, and deal with the experimental data by standard statistical and information-theoretical methods. These results may give rise to significant applications in quantum information processing such as quantum metrology. For closed systems, it is well known that uncertainty relation determines the precision limit of quantum metrology based on complementary basis. For open systems, with $B$ the environment and $A$ the system to be measured, our equality presents a complete characterization of the precision limit for measuring the system under MUBs. Such precision limit or accuracy determined by uncertainty relations also appear in quantum computing when quantum gates like CNOT are physically implemented. Hence our results may highlight further studies on both fundamental problems in quantum mechanics and the applications.

\section{METHODS}

A: Proof of the theorem

In a $d$-dimensional Hilbert space $\mathcal{H}$, let $\left\{\left|i_{\theta}\right\rangle \mid i=1, \cdots, d\right\}$ denote a basis labeled by $\theta$. A set of $M$ such bases is called mutually unbiased if

$\left|\left\langle i_{\theta} \mid j_{\tau}\right\rangle\right|^{2}=\frac{1}{d}$

for any $i, j=1,2, \cdots, d$, and $\theta, \tau=1, \cdots, M$ with $\theta \neq \tau$. When $d$ is a power of a 
prime number, a complete set of $d+1$ MUBs exists. When $d$ is an arbitrary integer number, the maximal number of MUBs is unknown. For example, when $d=6$, only three MUBs have yet been found. However, for any $d \geq 2$, there exist at least three MUBs. For the purpose of this paper, we only assume that $M$ MUBs are available in a $d$-dimensional Hilbert space, where $M$ is less than or equal to the maximal number of MUBs that can exist.

In a composite Hilbert space $\mathcal{H} \otimes \mathcal{H}$ of two qubits, we construct the following $M(d-1)+1$ states,

$|\Phi\rangle=\frac{1}{\sqrt{d}} \sum_{i=1}^{d}\left|i_{1}\right\rangle_{A} \otimes\left|i_{1}\right\rangle_{B}^{*}$

$\left|\phi_{\theta, k}\right\rangle=\frac{1}{\sqrt{d}} \sum_{i=1}^{d} \omega^{k(i-1)}\left|i_{\theta}\right\rangle_{A} \otimes\left|i_{\theta}\right\rangle_{B}^{*}$

with $\omega=e^{2 \pi i / d}, k=1, \ldots, d-1$ and $\theta=1,2, \ldots, M$. Here $\left|i_{\theta}\right\rangle^{*}$ denotes the complex conjugate of $\left|i_{\theta}\right\rangle$ with respect to the computational basis (which can be chosen as the first basis $\left\{\left|i_{1}\right\rangle\right\}$ without loss of generality). It is straightforward to show that the $M(d-1)+1$ bipartite states defined in Eqs. (10) and (11) are normalized and orthogonal to each other.

Therefore, these $M(d-1)+1$ states can be used for constructing a basis in the composite Hilbert space $\mathcal{H} \otimes \mathcal{H}$. Since there are at most $d^{2}$ orthogonal states in a $d^{2}$-dimensional space, one has $M(d-1)+1 \leq d^{2}$, which implies $M \leq d+1$, i.e., there are at most $d+1$ MUBs for a $d$ dimensional Hilbert space. When $M=d+1$, i.e., a complete set of $d+1$ MUBs in a $d$-dimensional space is available, the states defined in Eqs. (10) and (11) constitute a complete basis for the composite Hilbert space $\mathcal{H} \otimes \mathcal{H}$. On the other hand, when $M<d+1$, one can complete a basis of the composite Hilbert space by adding $p=(d-1)(d+1-M)$ additional orthonormal states $\left\{\left|\phi_{a}\right\rangle \mid a=1, \cdots, p\right\}$. The projector onto the subspace spanned by these additional states is denoted by $\mathcal{P}$, i.e.,

$\mathcal{P}=I \otimes I-|\Phi\rangle\left\langle\Phi\left|-\sum_{\theta=1}^{M} \sum_{k=1}^{d-1}\right| \phi_{\theta, k}\right\rangle\left\langle\phi_{\theta, k}\left|=\sum_{a=1}^{p}\right| \varphi_{a}\right\rangle\left\langle\varphi_{a}\right|$.

It is obvious that $\mathcal{P}=0$ when $M=d+1$.

Let $T_{2}$ denote the partial transpose with respect to the computational basis of the second Hilbert space. One immediately has

$(|\Phi\rangle\langle\Phi|)^{T_{2}}=\frac{1}{d} \sum_{i, j=1}^{d}\left|i_{1}\right\rangle\left\langle j_{1}|\otimes| j_{1}\right\rangle\left\langle i_{1}\right|$.

As $\sum_{k=1}^{d-1} \omega^{k(i-j)}$ equals to -1 for $i \neq j$ and $d-1$ when $i=j$, it is not difficult to show

$$
\left(\sum_{k=1}^{d-1}\left|\phi_{\theta, k}\right\rangle\left\langle\phi_{\theta, k}\right|\right)^{T_{2}}=\sum_{i=1}^{d}\left|i_{\theta}\right\rangle\left\langle i_{\theta}|\otimes| i_{\theta}\right\rangle\left\langle i_{\theta}\left|-\frac{1}{d} \sum_{i, j=1}^{d}\right| i_{\theta}\right\rangle\left\langle j_{\theta}|\otimes| j_{\theta}\right\rangle\left\langle i_{\theta}\right| .
$$

Suppose $\rho_{A B}$ is a bipartite state on the composite Hilbert space $\mathcal{H}_{A} \otimes \mathcal{H}_{B}$ of dimension $d \times D$, and suppose $\left\{\left|i_{\theta}\right\rangle_{A}\right\}$ is the $\theta$ th MUB in the $d$-dimensional Hilbert space $\mathcal{H}_{A}$, after system $A$ is projected onto the $\theta$ th MUB the overall bipartite state is written as

$\rho_{\theta B}=\sum_{i=1}^{d}\left|i_{\theta}\right\rangle_{A}\left\langle i_{\theta}\right| \otimes_{A}\left\langle i_{\theta}\left|\rho_{A B}\right| i_{\theta}\right\rangle_{A}$.

Given a bipartite state $\rho_{A B}$ and a set of $M$ MUBs in $\mathcal{H}_{A}$, we define an operator

$\Gamma_{A B} \equiv I_{A} \otimes \rho_{B}+\frac{M-1}{d} \rho_{A B}-\sum_{\theta=1}^{M} \rho_{\theta B}$

on $\mathcal{H}_{A} \otimes \mathcal{H}_{B}$. This operator is Hermitian, and it has the following nice property.

Proposition When $M=d+1$, the operator $\Gamma_{A B}$ vanishes: $\Gamma_{A B}=0$. When $M \leq d$, it is nonnegative-definite

$\Gamma_{A B} \geq 0$.

In order to prove the proposition, we introduce an additional Hilbert space $\mathcal{H}_{C}$ of dimension $d$, and introduce a linear map $\mathcal{F}$ that maps operators on $\mathcal{H}_{C}$ to operators on $\mathcal{H}_{A}$, such that $\mathcal{F}\left(\left|i_{1}\right\rangle_{C}\left\langle j_{1}\right|\right)=\left|i_{1}\right\rangle_{A}\left\langle j_{1}\right|(i, j=$ $1, \cdots, d)$. One can easily show that $\mathcal{F}\left(\left|i_{\theta}\right\rangle_{C}\left\langle j_{\theta}\right|\right)=\left|i_{\theta}\right\rangle_{A}\left\langle j_{\theta}\right|$ for $\theta=1, \ldots, M$. Let $\mathcal{F}^{-1}$ denote the inverse map, and let $\rho_{C B} \equiv \mathcal{F}^{-1}\left(\rho_{A B}\right)$ denote the corresponding state on $\mathcal{H}_{C} \otimes \mathcal{H}_{B}$ with respect to the state $\rho_{A B}$ on $\mathcal{H}_{A} \otimes \mathcal{H}_{B}$. Therefore, the map $\mathcal{F}: \rho_{C B} \rightarrow \mathcal{F}\left(\rho_{C B}\right)$ can also be conveniently written via a partial trace over system $C$

$$
\begin{gathered}
\mathcal{F}\left(\rho_{C B}\right)=\sum_{i j} \operatorname{Tr}_{C}\left\{\left[\left|i_{\theta}\right\rangle_{A}\left\langle j_{\theta}|\otimes| j_{\theta}\right\rangle_{C}\left\langle i_{\theta}\right|\right] \rho_{C B}\right\} \\
=\sum_{i j}\left|i_{\theta}\right\rangle_{A}\left\langle j_{\theta}\right| \otimes\left\langle i_{\theta}\left|\rho_{C B}\right| j_{\theta}\right\rangle \\
=\rho_{A B}
\end{gathered}
$$

for any $\theta \in\{1, \cdots, M\}$. Similarly, $\rho_{\theta B}$ can be written as

$$
\rho_{\theta B}=\operatorname{Tr}_{C}\left\{\left[\sum_{i=1}^{d}\left|i_{\theta}\right\rangle_{A}\left\langle i_{\theta}|\otimes| i_{\theta}\right\rangle_{C}\left\langle i_{\theta}\right|\right] \rho_{C B}\right\} \text {. }
$$

Hence

$$
\begin{aligned}
\sum_{\theta=1}^{M}\left(\frac{1}{d} \rho_{A B}\right. & \left.-\rho_{\theta B}\right)=\sum_{\theta=1}^{M} \operatorname{Tr}_{C}\left\{\frac{1}{d} \sum_{i j}\left|i_{\theta}\right\rangle_{A}\left\langle j_{\theta}|\otimes| j_{\theta}\right\rangle_{C}\left\langle i_{\theta}\right| \rho_{C B}\right\} \\
& -\sum_{\theta=1}^{M} \operatorname{Tr}_{C}\left\{\sum_{i}\left|i_{\theta}\right\rangle_{A}\left\langle i_{\theta}|\otimes| i_{\theta}\right\rangle_{C}\left\langle i_{\theta}\right| \rho_{C B}\right\} \\
= & -\operatorname{Tr}_{C}\left\{\left(\sum_{\theta=1}^{M} \sum_{k=1}^{d-1}\left|\phi_{\theta, k}\right\rangle_{A C}\left\langle\phi_{\theta, k}\right|\right)^{T_{C}} \rho_{C B}\right\} .
\end{aligned}
$$

We have used Eq. (14) to obtain the last equality. From Eqs. (13) and (18) with $\theta=1$, we also have

$$
\frac{1}{d} \rho_{A B}=\operatorname{Tr}_{C}\left\{\left(|\Phi\rangle_{A C}\langle\Phi|\right)^{T_{C}} \rho_{C B}\right\} \text {. }
$$

From Eqs. (20) and (21) and the obvious relation $I_{A} \otimes \rho_{B}=\operatorname{Tr}_{C}\left[\left(I_{a} \otimes I_{C}\right)\right.$ $\left.\rho_{C B}\right]$, we can rewrite $\Gamma_{A B}$ as

$$
\begin{gathered}
\Gamma_{A B}=I_{A} \otimes \rho_{B}-\frac{1}{d} \rho_{A B}+\sum_{\theta=1}^{M}\left(\frac{1}{d} \rho_{A B}-\rho_{\theta B}\right) \\
=\operatorname{Tr}_{C}\left\{\mathcal{P}_{A C}^{T_{C}} \rho_{C B}\right\} .
\end{gathered}
$$

Here the operator $\mathcal{P}_{A C}$ is the projector defined on $\mathcal{H}_{A} \otimes \mathcal{H}_{C}$ according to (12), i.e.,

$$
\begin{gathered}
\mathcal{P}_{A C}=I \otimes I-|\Phi\rangle\left\langle\Phi\left|-\sum_{\theta=1}^{M} \sum_{k=1}^{d-1}\right| \phi_{\theta, k}\right\rangle\left\langle\phi_{\theta, k}\right| \\
=\sum_{a=1}^{p}\left|\varphi_{a}\right\rangle_{A C}\left\langle\varphi_{a}\right| .
\end{gathered}
$$

When $M=d+1$, then $p=0$, the states $\left|\phi_{a}\right\rangle_{A C}$ in $\mathcal{H}_{A} \otimes \mathcal{H}_{C}$ do not exist, both $\mathcal{P}_{A C}$ and $\Gamma_{A B}$ vanish. When $M \leq d$, we have

$$
\begin{gathered}
\Gamma_{A B}=\operatorname{Tr}_{C}\left\{\sum_{a=1}^{p}\left(\left|\varphi_{a}\right\rangle_{A C}\left\langle\varphi_{a}\right|\right)^{T_{C}} \rho_{C B}\right\} \\
=\sum_{a=1}^{p} \operatorname{Tr}_{C}\left\{\left(\left|\varphi_{a}\right\rangle_{A C}\left\langle\varphi_{a}\right|\right)^{T_{C}} \rho_{C B}\right\} \\
=\sum_{a=1}^{p} \operatorname{Tr}_{C}\left\{\left(\left|\varphi_{a}\right\rangle_{A C}\right)^{T_{C}} \rho_{C B}\left(\left\langle\left.\varphi_{a}\right|_{A C}\right)^{T_{C}}\right\} .\right.
\end{gathered}
$$

The last equality is due to the fact that operators on $\mathcal{H}_{C}$ can have cyclic permutations under the partial trace over $C$. Let $\Theta_{a} \equiv\left(\left|\varphi_{a}\right\rangle_{A C}\right)^{T_{C}} \sqrt{\rho_{C B}}$, which are operators on $\mathcal{H}_{A} \otimes \mathcal{H}_{C} \otimes \mathcal{H}_{B}$. Then we have

$\Gamma_{A B}=\sum_{\alpha=1}^{p} \operatorname{Tr}_{C}\left\{\Theta_{a}\left(\Theta_{a}\right)^{\dagger}\right\}$.

Since $\Theta_{\alpha}\left(\Theta_{\alpha}\right)^{\dagger}$ are always nonnegative-definite operators on $\mathcal{H}_{A} \otimes \mathcal{H}_{C} \otimes \mathcal{H}_{B}$, the operators $\operatorname{Tr}_{C}\left\{\Theta_{\alpha}\left(\Theta_{a}\right)^{\dagger}\right\}$ are nonnegative-definite operators on $\mathcal{H}_{A} \otimes \mathcal{H}_{B}$, so is their sum. Therefore $\Gamma_{A B} \geq 0$. This completes the proof of the proposition.

According to the proposition, $\Gamma_{A B}$ is a nonnegative-definite operator when $M \leq d$, and it vanishes when $M=d+1$. Hence, for any nonnegativedefinite operator $\Pi_{A B}$ on $\mathcal{H}_{A} \otimes \mathcal{H}_{B}$

$\operatorname{Tr}_{A B}\left(\Gamma_{A B} \Pi_{A B}\right) \geq 0$

when $M \leq d$, and the inequality becomes an equality when $M=d+1$.

Let $\Pi_{A B}=\rho_{A B}$, Eq. (25) yields

$$
\begin{gathered}
0 \leq \operatorname{Tr}_{A B}\left(\Gamma_{A B} \rho_{A B}\right) \\
=\operatorname{Tr}\left(\rho_{B}^{2}\right)+\frac{M-1}{d} \operatorname{Tr}\left(\rho_{A B}^{2}\right)-\sum_{\theta=1}^{M} \operatorname{Tr}\left(\rho_{\theta B}^{2}\right) .
\end{gathered}
$$


Therefore,

$-\sum_{\theta=1}^{M} \operatorname{Tr}\left(\rho_{\theta B}^{2}\right) \geq-\operatorname{Tr}\left(\rho_{B}^{2}\right)-\frac{M-1}{d} \operatorname{Tr}\left(\rho_{A B}^{2}\right)$.

Adding $\operatorname{MTr}\left(\rho_{B}^{2}\right)$ to the above inequality, we immediately have

$\sum_{\theta=1}^{M}\left(\operatorname{Tr}\left(\rho_{B}^{2}\right)-\operatorname{Tr}\left(\rho_{\theta B}^{2}\right)\right) \geq(M-1)\left(\operatorname{Tr}\left(\rho_{B}^{2}\right)-\frac{1}{d} \operatorname{Tr}\left(\rho_{A B}^{2}\right)\right)$.

The inequality becomes an equality when $M=d+1$. Thus, the theorem in the main text has been proved.

Remark: The approach we admitted here is methodologically similar to the one used in ref. ${ }^{26}$ (see also ref. ${ }^{29}$ ). In ref. ${ }^{45}$ an elegant uncertainty equality has also been presented based on ONE positive operator-valued measure consisting uniformly all the measurement operators of $d+1$ MUBs. As we take into account all the $d+1$ MUB projection measurements individually, our uncertainty relation is completely different from the one given in ref. ${ }^{45}$ In fact, the uncertainty relation in ref. ${ }^{45}$ cannot be applied to our QRNG self-testing scenario, because the equality derived in ref. ${ }^{45}$ holds only when the system is measured in one of the MUBs with uniformly random probability. While our equality holds no matter whether the MUB is uniformly chosen. Such a property is crucial in practical QRNG application, as one needs to restrict the input randomness to choose a mutually unbiased basis.

\section{B: Quantum register}

We used the sample named 1-bromo-2,4,5-trifluorobenzene as a five-qubit NMR quantum system which consists of two ${ }^{1} \mathrm{H}$ spins and three ${ }^{19} \mathrm{~F}$ spins, dissolved in the liquid-crystal $\mathrm{N}$-(4-methoxybenzylidene)-4-butylaniline. The structure of the molecule is shown in Fig. 4. Due to the partial average effect in the liquid-crystal solution, the direct dipole-dipole interaction will be scaled down by the order parameter. ${ }^{61}$ In our sample, the partial average effect makes the direct dipole-dipole couplings of homonuclear spins much smaller than the difference between the chemical shifts of related nuclear spins, all the dipole-dipole Hamiltonians are reduced to the form of $\sigma_{z}^{i} \sigma_{z}^{j}$. Therefore, the effective Hamiltonian of the five-qubit system in rotating frame is

$H_{\mathrm{NMR}}=\sum_{i=1}^{5} \pi \nu_{i} \sigma_{z}^{i}+\sum_{1 \leq j<k \leq 5} \frac{\pi}{2}\left(J_{j k}+2 D_{j k}\right) \sigma_{z}^{j} \sigma_{z}^{k}$,

where $\sigma_{z}$ is the Pauli operator, $v_{i}$ is the chemical shift of spin- $i$ and $J_{j k}+2 D_{j k}$ is the effective coupling constant of spin- $j$ and spin- $k$. The relevant parameters are shown in Fig. 4.

\section{C: Initial state preparation}

We first initialized the quantum register into a labeled pseudo-pure state (LPPS) $\rho_{\text {LPPS }}=\varepsilon \sigma_{z}^{\text {probe }} \otimes|0000\rangle_{A B A B}\langle 0000|+1 / 32 l_{32}$ from the equilibrium state where $\varepsilon$ is the polarization about $10^{-5}$. We just neglected the identity part because unitary and nonunitary operations all have no influence on it. We applied 30 unitary operators with the form $U=e^{-i \beta_{i j} V_{i j}}$ to redistribute the populations between energy levels $i$ and $j$. Here $\mathcal{V}_{i j}$ is the single quantum transition operator between levels $i$ and $j$, that is, a $32 \times 32$ matrix whose elements are zero except for two elements $\mathcal{V}_{i j}(i, j)=\mathcal{V}_{i j}(j, i)=1 / 2$. According to the desired distribution, the angle $\beta_{i j}$ were numerically calculated. In this produce, the unitary operators generated undesired coherence terms that were eliminated by a following field gradient pulse $G z$. Accordingly, the LPPS $\rho_{\text {LPPS }}$ was prepared.

In the following we only consider the deviation part $\sigma_{z}^{\text {probe }} \otimes|0000\rangle_{A B A B B I}\langle 0000|$. The composite system was prepared into the state

$$
\begin{aligned}
\sigma_{z}^{\text {probe }} \otimes \rho_{A B}(a, x) \otimes & \rho_{A \prime B \prime}(a, x)=\sigma_{z}^{\text {probe }} \otimes\left(x\left|\psi_{a}\right\rangle\left\langle\psi_{a}\right|+\frac{1-x}{4} I_{4}\right) \\
& \otimes\left(x\left|\psi_{a}\right\rangle\left\langle\psi_{a}\right|+\frac{1-x}{4} I_{4}\right)
\end{aligned}
$$

from $\sigma_{z}^{\text {probe }} \otimes|0000\rangle\langle 0000|$, where $\left|\psi_{a}\right\rangle=\cos (\alpha / 2)|01\rangle-\sin (\alpha / 2)|10\rangle$. In the case of $x=1$, the bipartite systems are entangled where the rotation angle $a$ varies from 0 to $\pi / 2$ for producing different entanglement. $a=0$ corresponds to separable state and $a=\pi / 2$ corresponds to the maximally entangled state. Setting $a=\pi / 2$ and scanning $x$ from 0 to 1 with interval

\begin{tabular}{|c|c|c|c|c|c|c|c|c|}
\hline (a) & $F_{5}$ & $\mathrm{H}_{4}$ & $\mathbf{H}_{3}$ & $F_{2}$ & $F_{1}$ & & $T_{2}$ & $\mathrm{~T}_{1}$ \\
\hline & & 1915.7 & 63.5 & 342.5 & 144.4 & $F_{5}$ & $240 \mathrm{~ms}$ & $0.8 \mathrm{~s}$ \\
\hline & & 2492 & -12.8 & 1556.6 & 64.5 & $\mathbf{H}_{4}$ & $310 \mathrm{~ms}$ & $1.5 \mathrm{~s}$ \\
\hline & & & 2479 & 129.2 & 1887.4 & $\mathbf{H}_{3}$ & $313 \mathrm{~ms}$ & $1.5 \mathrm{~s}$ \\
\hline & & \multirow{2}{*}{\multicolumn{2}{|c|}{ Unit: $\mathrm{Hz}$}} & -44888 & -59.4 & $\mathbf{F}_{2}$ & $174 \mathrm{~ms}$ & $0.6 \mathrm{~s}$ \\
\hline & & & & & -47340 & $\mathbf{F}_{1}$ & $180 \mathrm{~ms}$ & $0.8 \mathrm{~s}$ \\
\hline
\end{tabular}
0.25 , we prepared the states with different mixedness. In preparing

(b)

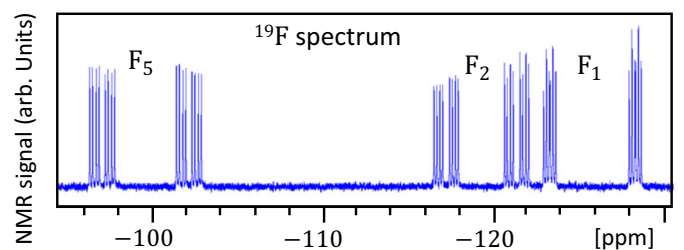

(c)

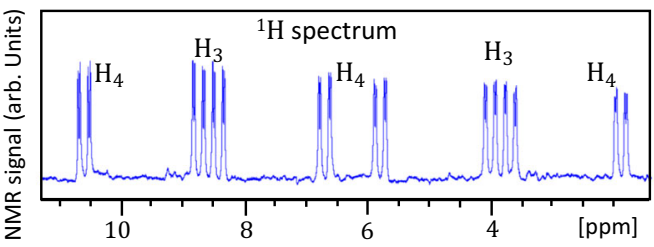

Fig. 4 a Characteristics of the 1-bromo-2,4,5-trifluorobenzene molecule. The chemical shifts and effective coupling constants (in $\mathrm{Hz}$ ) are on and above the diagonal in the table, respectively. The last two columns show the transversal relaxation times and the longitudinal relaxation times of each nucleus. b, c The thermal equilibrium ${ }^{19} \mathrm{~F}$ and ${ }^{1} \mathrm{H}$ spectra (a)

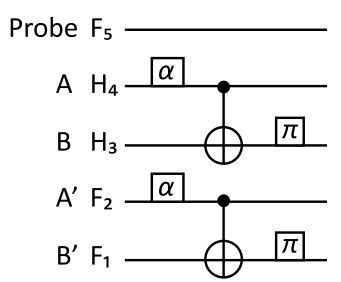

(b)

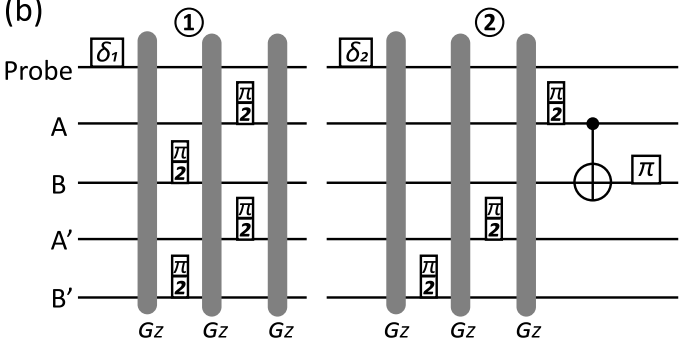

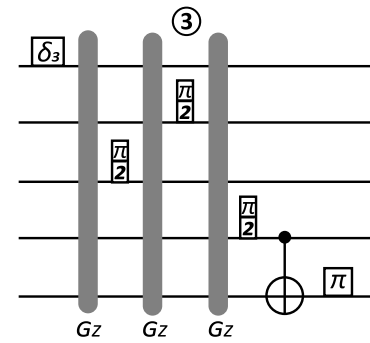

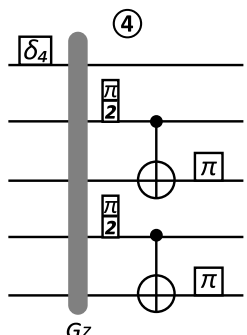

Fig. 5 a Quantum circuit of preparing $\sigma_{z}^{\text {probe }} \otimes \rho_{A B}(a, 1) \otimes \rho_{A \prime B /}(a, 1)$ from LPPS. The rectangles represent single-qubit rotations with angles $a$, $\pi / 2$ and $\pi$ along $y$-axis. $G z$ is the field gradient pulse along $z$-axis. b Pulse sequences for preparing $\sigma_{z}^{\text {probe }} \otimes \rho_{A B}(\pi / 2, x) \otimes \rho_{A / B \prime}(\pi / 2, x)$ with the temporal averaging method. The rotation angles $\delta_{1,2,3,4} \operatorname{are} \arccos \left[(1-x)^{2}\right], \arccos [x(1-x)], \arccos [x(1-x)]$ and $\arccos \left(x^{2}\right)$, which $\operatorname{are}$ used to adjust the coefficients of four parts in Eq. (30) 


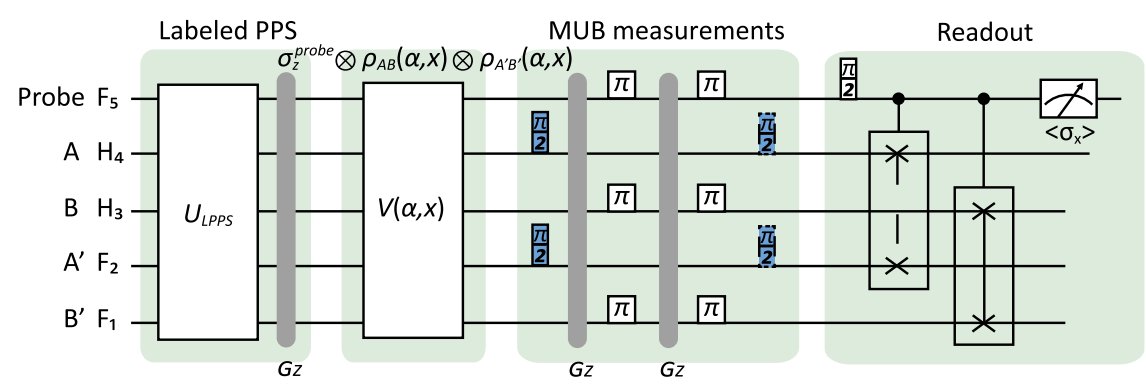

Fig. 6 Experimental pulse sequence. The LPPS preparation contains one GRAPE pulse and one $z$-axis gradient pulse. The GRAPE pulse is used to redistribute the populations and the field gradient pulse is used to eliminate the undesired coherences. The operation $V(a, x)$ is to prepare the initial state $\sigma_{z}^{\text {probe }} \otimes \rho_{A B}(a, x) \otimes \rho_{A^{\prime} B}(a, x)$. Its specific form is shown in Fig. 5 . The solid blue rectangle is $\pi / 2$ rotation along $(-y)$-axis in $x$ measurement and along $x$-axis in $y$-measurement. The dashed blue rectangles denote $\pi / 2$ rotations with the opposite phases to those in solid blue rectangles. No need to operate them in z-measurement. The readout part contains two $C_{\text {swap }}$ gates applying on subsystems $A A^{\prime}$ and $B B^{\prime}$. The dashed $C_{\text {swap }}\left(A A^{\prime}\right)$ is not required in reading out the purity of subsystem $B$

(a)
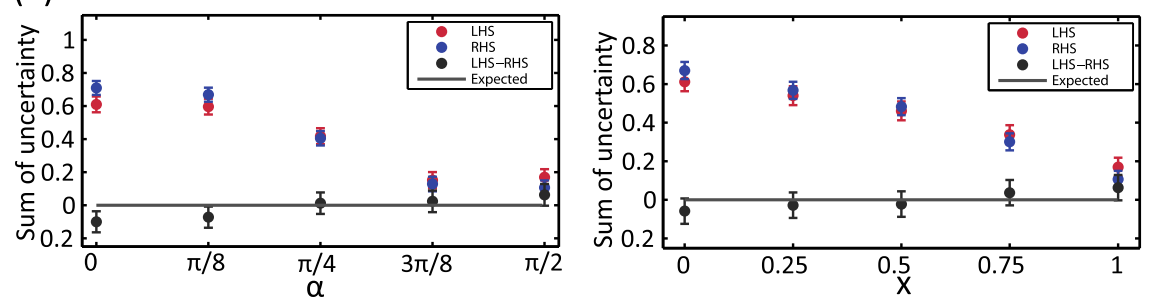

(b)
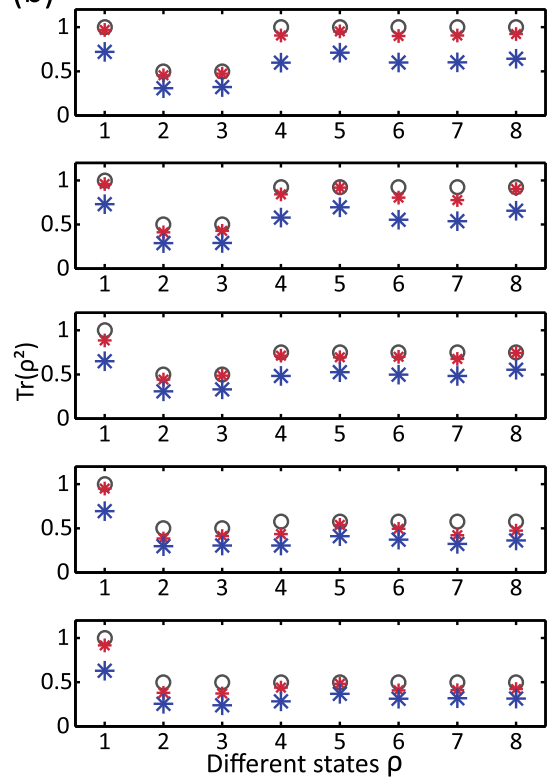
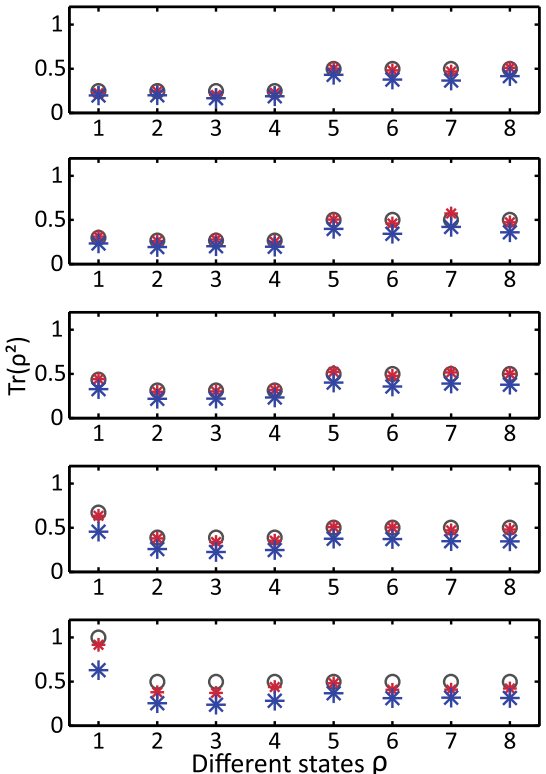

Fig. 7 a The original results without rescaling of verifying the measurement uncertainty relation. The bars are plotted from the infidelity of readout process. $\mathbf{b}$ The directly measured purities. The hollow dots are the expected purities. The blue asterisks are the directly measured purities and the red ones are the rescaled results. The left five figures correspond to purities when the initial bipartite state is $\rho_{A B}(a, 1)$ where $a$ $=0, \pi / 8, \pi / 4,3 \pi / 8, \pi / 2$ from top to bottom. And the right five figures correspond to $\rho_{A B}(\pi / 2, x)$ where $x=0,0.25,0.5,0.75,1$ from top to bottom. In each figure, from left to right, they are $\operatorname{Tr}\left(\rho_{A B}^{2}\right), \operatorname{Tr}\left(\rho_{x B}^{2}\right), \operatorname{Tr}\left(\rho_{y B}^{2}\right), \operatorname{Tr}\left(\rho_{z B}^{2}\right), \operatorname{Tr}\left(\rho_{B}^{2}\right), \operatorname{Tr}\left[\left(\rho_{B \mid x}\right)^{2}\right], \operatorname{Tr}\left[\left(\rho_{B \mid y}\right)^{2}\right], \operatorname{Tr}\left[\left(\rho_{B \mid z}\right)^{2}\right], w h e r e \rho_{\theta B}(\theta=x, y, z)$ are the resulting state after the complete MUB measurements on subsystem $A$, and $\rho_{B \mid \theta}=\operatorname{Tr}_{A}\left(\rho_{\theta B}\right)$

$\sigma_{z}^{\text {probe }} \otimes \rho_{A B}(\pi / 2, x) \otimes \rho_{A \prime B}(\pi / 2, x)$, we divided it into 4 parts, i.e., $\sigma_{z}^{\text {probe }} \otimes\left(\frac{1-x}{4} I_{2}\right) \otimes\left(\frac{1-x}{4} I_{2}\right)$,

$\sigma_{z}^{\text {probe }} \otimes\left(\frac{1-x}{4} I_{2}\right) \otimes\left(x\left|\psi_{\pi / 2}\right\rangle\left\langle\psi_{\pi / 2}\right|\right)$,

$\sigma_{z}^{\text {probe }} \otimes\left(x\left|\psi_{\pi / 2}\right\rangle\left\langle\psi_{\pi / 2}\right|\right) \otimes\left(\frac{1-x}{4} I_{2}\right)$,

$\sigma_{z}^{\text {probe }} \otimes\left(x\left|\psi_{\pi / 2}\right\rangle\left\langle\psi_{\pi / 2}\right|\right) \otimes\left(x\left|\psi_{\pi / 2}\right\rangle\left\langle\psi_{\pi / 2}\right|\right)$,

and adopted the temporal averaging $\operatorname{method}^{62}$ to realize them. By summing the four output states, we obtained the desired state $\sigma_{z}^{\text {probe }} \otimes \rho_{A B}(\pi / 2, x) \otimes \rho_{A^{\prime} B}(\pi / 2, x)$. The pulse sequences for the state preparation are shown in Fig. $5 a, b$.

D: Experimental procedure

The total experimental procedure is shown in Fig. 6, including the LPPS preparation, the initial state preparation, MUB measurements and the readout of the purities. When the measurement is along $y$-axis, the first and last rotations in the MUB measurement part should be substituted by 
rotations along $x$ and $-x$, and the angles remain also $\pi / 2$. In the $z$-axis measurement case, these two rotations are omitted.

\section{E: The attenuation factors caused by decoherence effect and experimental measured purities}

Even through the high-fidelity GRAPE pulses in experiments, the experimental results are still severely affected due to the decoherence due to the long running times comparing to the $T_{2}$ relaxation times of nuclear spins.

In our experiments, the environmental noises are suitably modeled as Markovian, and the evolution of the system is given by the Lindblad master equation

$\dot{\rho}=-i\left[H_{\mathrm{S}}+H_{\mathrm{C}}(t), \rho\right]+\sum_{\alpha}\left(2 L_{a} \rho L_{a}^{\dagger}-L_{a}^{\dagger} L_{\alpha} \rho-\rho L_{a}^{\dagger} L_{\alpha}\right)$,

where $H_{\mathrm{S}}$ is the system Hamiltonian, $H_{\mathrm{C}}(t)$ is the time-dependent external control Hamiltonian, and the operators $L_{a}$ are Lindblad operators representing the coupling with the environment. The experiments are mainly affected by the dephasing process, and therefore $L_{a}=\sqrt{\frac{Y_{a}}{2}} \sigma_{z}^{a},(a=$ $1, \ldots, 5)$ and $\gamma_{a}=1 / T_{2 a}$ and

$\dot{\rho}=-i\left[H_{\mathrm{S}}+H_{\mathrm{C}}(t), \rho\right]+\sum_{a} \gamma_{a}\left(\sigma_{z}^{a} \rho \sigma_{z}^{a}-\rho\right)$.

In the experiments, the high-fidelity GRAPE pulses we used are kinds of shaped pulses consisting of thousands of slices with a constant $H_{C}\left(t_{i}\right)$ the duration of each slice, where $\delta t$ is $2-25 \mu$ s. Consequently, for each slice, the state of the system can be approximately given by

$\rho\left(t_{i+1}\right)=\rho\left(t_{i}+\delta t\right)=\sum_{a=1}^{5} \sum_{i=0}^{1} E_{i}^{a} e^{-i\left[H_{s}+H_{c}\left(t_{i+1}\right)\right] \delta t} \rho\left(t_{i}\right) e^{i\left[H_{s}+H_{c}\left(t_{i+1}\right)\right] \delta t} E_{i}^{a \dagger}$,

where the Kraus operations

$E_{0}^{\alpha}=\sqrt{\lambda_{\alpha}} \mathbf{1}, E_{1}^{\alpha}=\sqrt{1-\lambda_{\alpha}} \sigma_{z}^{\alpha}$

with $\lambda_{a}=\left(1+e^{-\gamma_{a} \delta t}\right) / 2$.

Therefore, we numerically simulated the experiments, extracted the attenuation factors caused by decoherence effect and then rescaled the experiment results for verifying the uncertainty conversation relation in Fig. 3a, b. The un-rescaled original results are shown in Fig. 7a. From this we can see that even though the right and left sides of the uncertainty equation are all reduced, they almost equal each other. Figure $7 \mathrm{~b}$ shows the directly measured purities of different states required in the uncertainty conversation relation.

\section{DATA AVAILABILITY}

The main data supporting the finding of this study are available within the article. Additional data can be provided upon request.

\section{ACKNOWLEDGEMENTS}

We thank Marcin Pawłowski, Simone Severini and Dawei Lu for helpfull discussions. This work was supported by the National Key R\&D Program of China (Grant No. 2018YFA0306600, 2016YFA0301801, 2017YFA0303703), National Natural Science Foundation of China (Grants No. 11425523, 11661161018, 11675113, 11605153, $11571313,11575173)$, Natural Science Foundation of Zhejiang province (Grants No. LQ19A050001), Anhui Initiative in Quantum Information Technologies (Grant No. AHY050000), and the Key Project of Beijing Municipal Commission of Education under No. KZ201810028042.

\section{AUTHOR CONTRIBUTIONS}

All authors researched, collated, and wrote this paper.

\section{ADDITIONAL INFORMATION}

Competing interests: The authors declare no competing interests.

Publisher's note: Springer Nature remains neutral with regard to jurisdictional claims in published maps and institutional affiliations.

\section{REFERENCES}

1. Heisenberg, W. Über den anschaulichen Inhalt der quantentheoretischen Kinematik und Mechanik. Z. Phys. 43, 172-198 (1927).

2. Robertson, H. P. The uncertainty principle. Phys. Rev. 34, 163 (1929).

3. Schrödinger, E. About Heisenberg uncertainty relation. Sitz. Preuss. Akad. Wiss. Phys. Math. Kl. 19, 296 (1930).

4. Maassen, H. \& Uffink, J. B. M. Generalized entropic uncertainty relations. Phys. Rev. Lett. 60, 1103 (1988).

5. Ozawa, M. Uncertainty relations for noise and disturbance in generalized quantum measurements. Ann. Phys. 311, 350 (2004).

6. Wu, S., Yu, S. \& Mølmer, K. Entropic uncertainty relation for mutually unbiased bases. Phys. Rev. A 79, 022104 (2009).

7. Branciard, C. Error-tradeoff and error-disturbance relations for incompatible quantum measurements. Proc. Natl Acad. Sci. USA 110, 6742 (2013).

8. Friedland, S., Gheorghiu, V. \& Gour, G. Universal uncertainty relations. Phys. Rev. Lett. 111, 230401 (2013).

9. Puchała, Z., Rudnicki, Ł. \& Życzkowski, K. Majorization entropic uncertainty relations. J. Phys. A: Math. Theor. 46, 272002 (2013).

10. Vallone, G., Marangon, D., Tomasin, M. \& Villoresi, P. Quantum randomness certified by the uncertainty principle. Phys. Rev. A 90, 052327 (2014).

11. Maccone, L. \& Pati, A. K. Stronger uncertainty relations for all incompatible observables. Phys. Rev. Lett. 113, 260401 (2014).

12. Busch, P., Lahti, P. \& Werner, R. F. Quantum root-mean-square error and measurement uncertainty relations. Rev. Mod. Phys. 86, 1261 (2014).

13. Kaniewski, J., Tomamichel, M. \& Wehner, S. Entropic uncertainty from effective anticommutators. Phys. Rev. A 90, 012332 (2014).

14. Horodecki, R., Horodecki, P., Horodecki, M. \& Horodecki, K. Quantum entanglement. Rev. Mod. Phys. 81, 865 (2009).

15. Berta, M., Christandl, M., Colbeck, R., Renes, J. M. \& Renner, R. The uncertainty principle in the presence of quantum memory. Nat. Phys. 6, 659 (2010).

16. Tomamichel, M. \& Renner, R. The uncertainty relation for smooth entropies. Phys. Rev. Lett. 106, 110506 (2011)

17. Tomamichel, M., Lim, C. C. W., Gisin, N. \& Renner, R. Tight finite-key analysis for quantum cryptography. Nat. Commun. 3, 634 (2012).

18. Gehring, T. et al. Implementation of continuous-variable quantum key distribution with composable and one-sided-device-independent security against coherent attacks. Nat. Commun. 6, 8795 (2015).

19. Bradler, K., Mirhosseini, M., Fickler, R., Broadbent, A. \& Boyd, R. Finite-key security analysis for multilevel quantum key distribution. New J. Phys. 18, 073030 (2016).

20. Wootters, W. K. \& Fields, B. D. Optimal state-determination by mutually unbiased measurements. Ann. Phys. (NY) 191, 363 (1989).

21. Klappenecker, A. \& Rötteler, M. Mutually unbiased bases are complex projective 2-designs. In Proc. 2005 IEEE International Symposium on Information Theory (ISIT 2005) 1740-1744. (IEEE Press, New York, 2005).

22. Durt, T., Englert, B. G., Bengtsson, I. \& Życzkowski, K. On mutually unbiased bases. Int. J. Quant. Inf. 8, 535-640 (2010).

23. Kalev, A. \& Gour, G. Mutually unbiased measurements in finite dimensions. New J. Phys. 16, 053038 (2014)

24. Zhu, H. Mutually unbiased bases as minimal Clifford covariant 2-designs. Phys. Rev. A 91, 060301 (2015).

25. Englert, B. G. Fringe visibility and which-way information: an inequality. Phys. Rev. Lett. 77, 2154 (1996).

26. Brukner, Č. \& Zeilinger, A. Operationally Invariant Information in quantum measurements. Phys. Rev. Lett. 83, 3354 (1999).

27. Spengler, C., Huber, M., Brierley, S., Adaktylos, T. \& Hiesmayr, B. C. Entanglement detection via mutually unbiased bases. Phys. Rev. A 86, 022311 (2012).

28. Lu, D. et al. Tomography is necessary for universal entanglement detection with single-copy observables. Phys. Rev. Lett. 116, 230501 (2016).

29. Brukner, C. \& Zeilinger, A. Conceptual inadequacy of the Shannon information in quantum measurements. Phys. Rev. A 63, 022113 (2001).

30. Zeilinger, A. A foundational principle for quantum mechanics. Found. Phys. 29, 631 (1999).

31. Řeháček, J. \& Hradil, Z. Invariant information and quantum state estimation. Phys. Rev. Lett. 88, 130401 (2002)

32. Lee, J., Kim, M. S. \& Brukner, Č. Operationally invariant measure of the distance between quantum states by complementary measurements. Phys. Rev. Lett. 91 087902 (2003).

33. Kofler, J. \& Zeilinger, A. Quantum information and randomness. Eur. Rev. 18, 469 (2010).

34. Madhok, V., Riofro, C. A., Ghose, S. \& Deutsch, I. H. Information gain in tomography-a quantum signature of chaos. Phys. Rev. Lett. 112, 014102 (2014).

35. Lee, J. \& Kim, M. S. Entanglement teleportation via Werner states. Phys. Rev. Lett. 84, 4236 (2000).

36. Giovannetti, V., Lloyd, S. \& Maccone, L. Quantum-enhanced measurements: beating the standard quantum limit. Science 306, 1330-1336 (2004). 
37. Brukner, Č. \& Zeilinger, A. Young's experiment and the finiteness of information. Philos. Trans. R. Soc. Lond. A 360, 1061 (2002).

38. Luis, A. Complementarity and duality relations for finite-dimensional systems. Phys. Rev. A 67, 032108 (2003).

39. Song, W. \& Chen, Z.-B. Invariant information and complementarity in highdimensional states. Phys. Rev. A 76, 014307 (2007).

40. Brukner, C. \& Zeilinger, A. Information invariance and quantum probabilities. Found. Phys. 39, 631 (2009).

41. Rastegin, A. E. On the Brukner-Zeilinger approach to information in quantum measurements. Proc. R. Soc. A 471, 20150435 (2015).

42. Khrennikov, A. Reflections on Zeilinger-Brukner information interpretation of quantum mechanics. Found. Phys. 46, 836 (2016).

43. Pechen, A., Il'in, N., Shuang, F. \& Rabitz, H. Quantum control by von Neumann measurements. Phys. Rev. A 74, 052102 (2006).

44. Kalev, A., Mann, A. \& Revzen, M. Choice of measurement as the signal. Phys. Rev. Lett. 110, 260502 (2013).

45. Berta, M., Coles, P. J. \& Wehner, S. Entanglement-assisted guessing of complementary measurement outcomes. Phys. Rev. A 90, 062127 (2014).

46. Li, H.-W., Pawłowski, M., Yin, Z.-Q., Guo, G.-C. \& Han, Z.-F. Semi-deviceindependent randomness certification using $n \rightarrow 1$ quantum random access codes. Phys. Rev. A 85, 052308 (2012).

47. Cao, Z., Zhou, H. \& Ma, X. Loss-tolerant measurement-device-independent quantum random number generation. New J. Phys. 17, 125011 (2015).

48. Cao, Z., Zhou, H., Yuan, X. \& Ma, X. Source-independent quantum random number generation. Phys. Rev. X 6, 011020 (2016).

49. Pawłowski, M. \& Żukowski, M. Entanglement-assisted random access codes. Phys. Rev. A 81, 042326 (2010).

50. Li, C.-F., Xu, J.-S., Xu, X.-Y., Li, K. \& Guo, G.-C. Experimental investigation of the entanglement-assisted entropic uncertainty principle. Nat. Phys. 7, 752-756 (2011).

51. Prevedel, R., Hamel, D. R., Colbeck, R., Fisher, K. \& Resch, K. J. Experimental investigation of the uncertainty principle in the presence of quantum memory. Nat. Phys. 7, 757-761 (2011).

52. Paris, M. G. A. \& Řeháček, J. (eds) Quantum State Estimation. Lecture Notes in Physics, Vol. 649 (Springer, New York, 2004).
53. Ekert, A. K., Alves, C. M. \& Oi, D. K. L. Direct estimations of linear and nonlinear functionals of a quantum state. Phys. Rev. Lett. 88, 21 (2002).

54. Peng, X. et al. Preparation of pseudo-pure states by line-selective pulses in nuclear magnetic resonance. Chem. Phys. Lett. 340, 509-516 (2001).

55. Knill, E., Laflamme, R., Martinez, R. \& Tseng, C.-H. An algorithmic benchmark for quantum information processing. Nature 404, 368-370 (2000).

56. Teklemariam, G., Fortunato, E. M., Pravia, M. A., Havel, T. F. \& Cory, D. G. NMR analog of the quantum disentanglement eraser. Phys. Rev. Lett. 86, 5845 (2001).

57. Levitt, M. Spin Dynamics (Wiley: West Sussex, England, 2001).

58. Khaneja, N., Reiss, T., Kehlet, C., Schulte-Herbruggen, T. \& Glaser, S. J. Optimal control of coupled spin dynamics: design of NMR pulse sequences by gradient ascent algorithms. J. Magn. Reson. 172, 296 (2005).

59. Vandersypen, L. M. K. et al. Experimental realization of Shor's quantum factoring algorithm using nuclear magnetic resonance. Nature 414, 883-887 (2001).

60. Vandersypen, L. M. K. Experimental quantum computation with nuclear spins in liquid solution. Preprint at https://arxiv.org/abs/quant-ph/0205193 (2001).

61. Dong, R. Y. Nuclear Magnetic Resonance of Liquid Crystals (Springer, New York, 1997).

62. Knill, E., Chuang, I. \& Laflamme, R. Effective pure states for bulk quantum computation. Phys. Rev. A. 57, 3348 (1998).

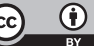

Open Access This article is licensed under a Creative Commons Attribution 4.0 International License, which permits use, sharing, adaptation, distribution and reproduction in any medium or format, as long as you give appropriate credit to the original author(s) and the source, provide a link to the Creative Commons license, and indicate if changes were made. The images or other third party material in this article are included in the article's Creative Commons license, unless indicated otherwise in a credit line to the material. If material is not included in the article's Creative Commons license and your intended use is not permitted by statutory regulation or exceeds the permitted use, you will need to obtain permission directly from the copyright holder. To view a copy of this license, visit http://creativecommons. org/licenses/by/4.0/.

(c) The Author(s) 2019 